\title{
Extracellular purines are biomarkers of neutrophilic airway inflammation
}

\author{
C.R. Esther Jr*, N.E. Alexis”, M.L. Clas*, E.R. Lazarowski ${ }^{\top}$, S.H. Donaldson`, \\ C.M. Pedrosa Ribeiro", C.G. Moore ${ }^{+}$, S.D. Davis* and R.C. Boucher ${ }^{\star}$
}

ABSTRACT: Purinergic signalling regulates airway defence mechanisms, suggesting that extracellular purines could serve as airway inflammation biomarkers in cystic fibrosis (CF).

The purines adenosine triphosphate (ATP), adenosine diphosphate (ADP), adenosine monophosphate (AMP) and adenosine were measured in sputum from 21 adults (spontaneously expectorated from seven CF patients, induced from 14 healthy controls) to assess normal values and CF-associated changes. Subsequently, purine levels were measured in bronchoalveolar lavage fluid (BALF) from 37 children (25 CF patients, 12 disease controls) and compared with neutrophil counts, presence of airway infection and lung function. To noninvasively assess airway purines, ATP levels were measured using luminometry in exhaled breath condensate (EBC) from 14 children with $\mathrm{CF}$ and 14 healthy controls, then 14 CF children during a pulmonary exacerbation.

Both ATP and AMP were elevated in sputum and BALF from CF subjects compared with controls. In BALF, ATP and AMP levels were inversely related to lung function and strongly correlated with neutrophil counts. In EBC, ATP levels were increased in CF relative to controls and decreased after treatment of CF pulmonary exacerbation.

The purines adenosine triphosphate and adenosine monophosphate are candidate biomarkers of neutrophilic airways inflammation. Measurement of purines in sputum or exhaled breath condensate may provide a relatively simple and noninvasive method to track this inflammation.

KEYWORDS: Biomarker, bronchoalveolar lavage fluid, cystic fibrosis, exhaled breath condensate, inflammation, purinergic signalling

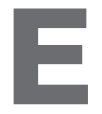
xtracellular adenyl purines, including the purine nucleotide adenosine triphosphate (ATP) and its metabolites, are important signalling molecules on airway surfaces. These purines serve as agonists for purinergic signalling pathways that play critical roles in airway mucus clearance. Indeed, ATP-mediated signalling regulates airway surface liquid volume, ciliary function and mucin secretion [1-3], each critical for control of mucus clearance rates.

Purines also mediate multiple components of inflammatory cell responses that are part of lung defence. For example, pro-inflammatory stimuli enhance release of ATP from epithelial [4-6] and inflammatory cells [7-10]. In particular, stimulated neutrophils release ATP and accumulate both extracellular ATP and adenosine monophosphate (AMP) [10, 11]. Inflammation also affects airway-luminal extracellular purine metabolism, both by altering ectonucleotidase activity on epithelial cell surfaces [12] and by addition of ectonucleotidase activity associated with the accumulation of inflammatory cells [8]. ATP released onto airway surfaces in response to inflammation also stimulates inflammatory cell responses, including chemotaxis and degranulation in neutrophils [10,13-15], cytokine production and oxidative bursts in macrophages [16-18], and activation of lymphocytes and eosinophils [17]. Adenosine formed as a consequence of ATP release also acts as a signalling molecule with both pro-inflammatory and anti-inflammatory effects $[19,20]$.

The importance of purinergic signalling in lung inflammation has been demonstrated in vivo. For example, ATP receptor ( $\mathrm{P}^{2} \mathrm{Y}_{1}-\mathrm{R}$ and $\left.\mathrm{P}_{2} \mathrm{Y}_{2}-\mathrm{R}\right)$ deficient mice exhibit impaired inflammatory responses and difficulty eradicating Pseudomonas from the lung [21]. Similarly, mouse strains with modest increases in lung adenosine have enhanced interleukin (IL)-4-mediated inflammatory responses [22], and higher lung
AFFILIATIONS

*Pediatric Pulmonology,

${ }^{*}$ Center for Environmental Medicine, Asthma and Lung Biology (CEMALB), "Cystic Fibrosis (CF) Research Center, and

${ }^{+}$Division of General Medicine and Epidemiology, University of North Carolina at Chapel Hill, Chapel Hill, NC, USA.

CORRESPONDENCE

C.R. Esther Jr

Pediatric Pulmonology, $5^{\text {th }}$ Floor Bioinformatics, CB\#7220, University of North Carolina at Chapel Hill, Chapel Hill, NC 27599-7220, USA. Fax: 19199666179

E-mail: Charles_Esther@

med.unc.edu

Received:

July 162007

Accepted after revision:

January 202008

\section{SUPPORT STATEMENT}

C.R. Esther Jr was supported by the National Institutes of Health (NIH), grant No. K12 RR17667, and the University of North Carolina Program for Translational Science. N.E. Alexis was supported by the US Environmental Protection Agency, grant No. CR829522. S.H. Donaldson was supported by grant №. K08 HL68617 from the NIH. C.G. Moore was supported by $\mathrm{NIH}$ grants $\mathrm{K} 12$ RR023248 and K30 RR022267. R.C. Boucher was supported by $\mathrm{NIH}$ grants HL34322 and HL084934. N.E. Alexis, E.R. Lazarowski, S.H. Donaldson and R.C. Boucher were supported by the NIH Specialized Centers of Clinically Oriented Research (SCCOR) program, grant No. NHLBI P5OHL084934.

STATEMENT OF INTEREST

None declared. 
adenosine levels lead to airway inflammation and pulmonary fibrosis [23, 24]. In vivo studies of airway purines in humans are limited, although increased levels of ATP have been observed in nasal lavage fluid [25] and blood [26] of subjects with cystic fibrosis (CF), and adenosine levels in airway secretions are elevated in untreated asthma and correlate with disease state [27-29].

The role of purines as mediators of the inflammatory response suggests that they may also be markers of inflammation. However, the concentrations and pattern of extracellular adenyl purines in the normal and diseased human airways surface remain largely unexplored. The aim of the present study was to measure the levels of purines in human airway secretions and evaluate their potential as biomarkers of airway inflammation, particularly in children with CF. First, purine levels were measured in sputum to establish normal values and assess changes associated with CF. In addition, purine levels were measured in the supernatant of mucopurulent material (SMM) aspirated from CF lungs removed for transplantation. Following this, bronchoalveolar lavage fluid (BALF) was collected from children undergoing clinically indicated bronchoscopy, and correlations were sought between purines and established markers of airways disease, including neutrophil counts, presence of infection and lung function. Finally, a simple and noninvasive method to measure airway purines in children by measuring ATP levels in exhaled breath condensate (EBC) was explored.

\section{METHODS AND MATERIALS \\ Study subjects}

Subject demographics are outlined in table 1. Control populations were healthy individuals, except in the BALF study,

\begin{tabular}{lcc} 
TABLE 1 Study subject demographics & \\
& CF & Control \\
\hline & & \\
Sputum study & & \\
Subjects (M/F) $n$ & $14(6 / 8)$ \\
Age yrs & $26.6 \pm 7.1$ & $26.9 \pm 5.4$ \\
FEV $1 \%$ pred & $28.3 \pm 11.6^{\#}$ & $103.4 \pm 9.5$ \\
BALF study & & \\
Subjects (M/F) $n$ & $25(11 / 14)$ & $12(7 / 5)$ \\
Age yrs & $10.5 \pm 3.7$ & $8.9 \pm 3.5$ \\
FEV $\%$ pred & $76.6 \pm 21.4^{\circ}$ & $104.3 \pm 24.4^{\circ}$ \\
EBC study & & $14(6 / 8)$ \\
Subjects (M/F) $n$ & $14(5 / 9)$ & $11.9 \pm 2.8$ \\
Age yrs & $13.8 \pm 3.3$ & $\mathrm{ND}$ \\
FEV $\%$ pred & $77.0 \pm 26.6^{+}$ & \\
EBC antibiotic study & & \\
Subjects (M/F) $n$ & $14(6 / 8)$ & \\
Age yrs & $14.8 \pm 3.5$ & \\
FEV $\%$ pred & $60.1 \pm 20.1$ & \\
\hline
\end{tabular}

Data are presented as mean $\pm \mathrm{SD}$, unless otherwise stated. CF: cystic fibrosis; M: male; F: female; FEV1: forced expiratory volume in one second; \% pred: \% predicted; BALF: bronchoalveolar lavage fluid; EBC: exhaled breath condensate; ND: not determined. \#: not available for three subjects; ": not available for four subjects; ${ }^{+}$: not available for one subject. which included the following disease controls: two subjects with primary ciliary dyskinesia and 10 subjects with recurrent cough or wheeze, all of whom were clinically stable at the time of bronchoscopy. All subjects were studied at the University of North Carolina at Chapel Hill (Chapel Hill, NC, USA), and studies were approved by the Institutional Review Board.

\section{Study design}

Differences between purine levels in airway secretions were assessed by comparison of CF with control populations. The relationship between purine levels and other markers of disease were assessed by regression analysis.

\section{Methods}

Sputum from healthy controls was collected and processed using induction as previously described [30]. Briefly, mucus plugs were selected, weighed and incubated with $0.1 \%$ dithiotreitol to solubilise mucus. Sodium citrate was added at $0.32 \%$ to the sample buffers to limit hydrolysis of purines. Samples were then washed in Dulbecco's PBS, filtered and analysed for cell viability, cell counts and differential. Sputum from CF subjects was collected by spontaneous expectoration, but was processed identically to induced samples.

SMM was recovered from the airway lumen of 13 excised human CF lungs at the time of transplant [31]. This material was centrifuged at $440,000 \times g$ for $60 \mathrm{~min}$ at $4{ }^{\circ} \mathrm{C}$ and the supernatant filtered through a $0.2-\mu \mathrm{m}$ filter and frozen at $-80^{\circ} \mathrm{C}$.

BALF was obtained via clinically indicated bronchoscopy. Aliquots were placed on ice, centrifuged at $11,000 \times g$ for $5 \mathrm{~min}$ at $4^{\circ} \mathrm{C}$ to remove cells and bacteria, and the supernatant immediately frozen and stored at $-80^{\circ} \mathrm{C}$. Separate aliquots were processed for cell differential and quantitative microbiological culture.

EBC was collected using an RTube ${ }^{\mathrm{TM}}$ device (Respiratory Research, Inc., Charlottesville, VA, USA). The chiller tube was held at $-10^{\circ} \mathrm{C}$ until immediately before the collection, and the subject exhaled through the device during $7 \mathrm{~min}$ of tidal breathing. No nose clips were used. EBC was recovered from the RTube ${ }^{\mathrm{TM}}$ and frozen at $-80^{\circ} \mathrm{C}$ until analysis.

\section{Purine analysis}

Adenyl purines were measured in airway secretions using etheno-derivatisation and HPLC [32]. Samples were boiled for 2 min prior to analysis, to inactivate nucleotidases.

\section{Luminometry}

The luciferin-luciferase assay was a modification of a previously described protocol [33]. In brief, $100 \mu \mathrm{L}$ aliquots from each sample were analysed in the light chamber of an LB953 AutoLumat luminometer (Berthold Technologies $\mathrm{GmbH}$, Bad Wildbad, Germany) after a $100 \mu \mathrm{L}$ injection of a luciferin-luciferase cocktail (luciferin $160 \mu \mathrm{g} \cdot \mathrm{mL}^{-1}$ and luciferase $8 \mu \mathrm{M}$ ). Luminescence was recorded for $10 \mathrm{~s}$ and compared with an ATP calibration curve performed in parallel.

\section{Analysis}

All data are expressed as mean $\pm \mathrm{SE}$, except demographic information, which is reported as mean \pm SD. Data that did not follow a normal distribution by D'Agostino-Pearson tests were log-transformed prior to analysis, including all purine 
measurements and neutrophil counts. Comparisons between groups were performed using unpaired t-tests, except for preand post-antibiotic comparisons, which were performed on paired data. Correlations were performed using Pearson's correlation.

\section{RESULTS}

\section{Purine levels in normal induced sputum and CF sputum}

To establish normal purine levels and assess whether these were altered in $\mathrm{CF}$, purines were measured in sputum collected by induction from 14 healthy adults and spontaneously expectorated from seven adult CF subjects. Given the relationship between purines and inflammation, it was hypothesised that sputum purines would be elevated in subjects with $\mathrm{CF}$, a disease characterised by high levels of airway inflammation. Purines were measured using ethenoderivatisation and HPLC, a technique which has been successfully utilised to measure purines from a variety of biological fluids [32]. Assessment of recovery and metabolism of purines in sputum revealed that purines could be readily
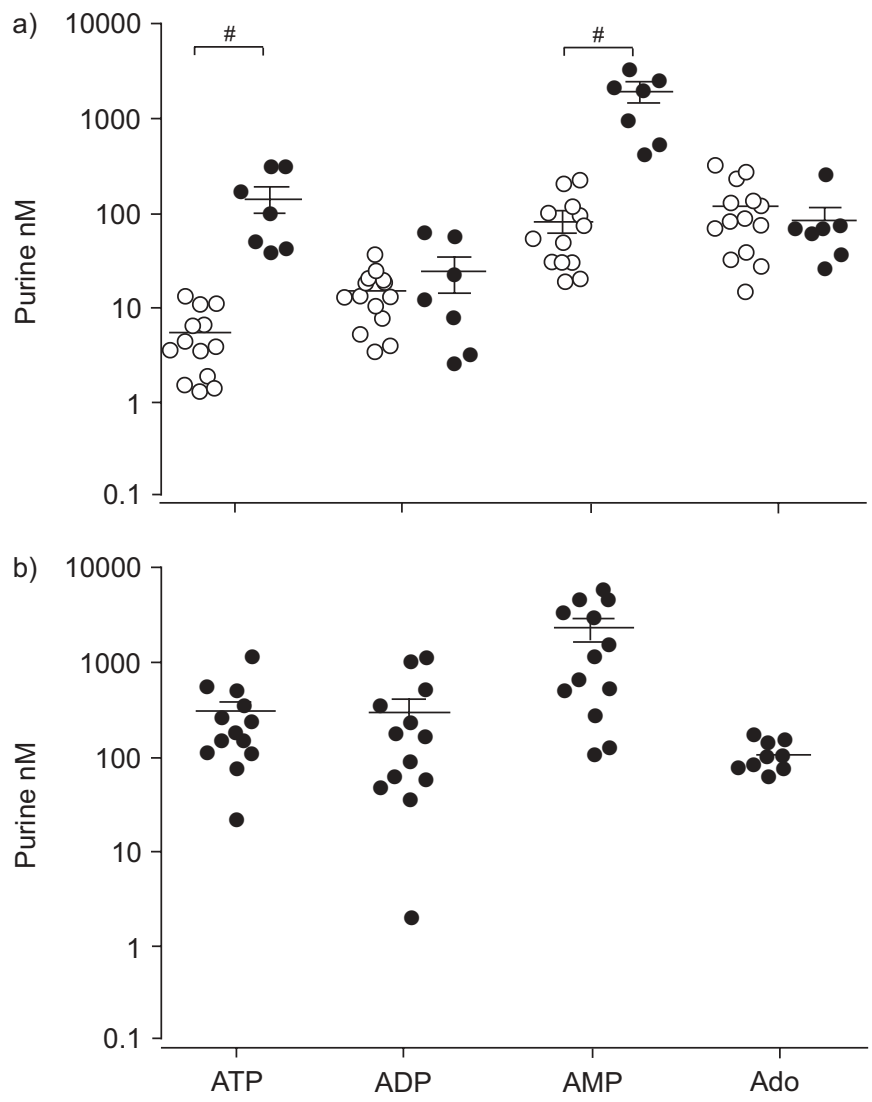

FIGURE 1. Airway purines were elevated in cystic fibrosis (CF). a) Sputum was obtained by spontaneous expectoration from adults with $C F(n=7 ; 0)$ and after induction in healthy controls $(n=14 ; 0)$, and purines measured in isolated supernatants. Both adenosine triphosphate (ATP) and adenosine monophosphate (AMP) were significantly elevated in CF compared with control, while adenosine diphosphate (ADP) and adenosine (Ado) levels were similar between groups. b) Supernatant of mucopurulent material (SMM) was obtained from the explanted lungs of adults with end-stage CF at time of lung transplantation $(n=13)$. The pattern of purine levels in SMM was similar to that observed in CF sputum. \# : $p<0.0001$ recovered and measured in both control and $\mathrm{CF}$ sputa (recovery rates: 89 and $81 \%$ for control and CF, respectively), and that purine metabolism during processing could be limited by addition of sodium citrate to the sample (for further details see supplementary data).

Analysis of sputa revealed the expected increase in neutrophilic inflammation in the CF sputa compared with control $\left(14,700\right.$ versus 240 neutrophils $\cdot \mathrm{mg}^{-1}$ sputum, respectively; $\mathrm{p}=0.01$ ). Differences in the pattern and concentration of purines were also observed. Analysis of purine concentrations in normal sputa revealed a pattern of adenosine $\approx \mathrm{AMP}>$ adenosine diphosphate (ADP) $>$ ATP. In contrast, CF sputa contained increased levels of both ATP and AMP, but not ADP or adenosine, relative to controls (fig. 1a). Indeed, the ATP to adenosine ratio was elevated in sputa from CF subjects compared with control (mean \pm SE: $33.0 \pm 6.6$ versus $0.99 \pm 0.2$, respectively; $\mathrm{p}=0.003$ ), as was the AMP to adenosine ratio $(1.85 \pm 0.4$ versus $0.08 \pm 0.0$ for $\mathrm{CF}$ and control, respectively; $p=0.007)$. To ensure that the induction procedure in controls did not affect the results, purine levels were examined in paired spontaneously expectorated and induced sputum samples from three individuals (one CF patient and two with chronic obstructive pulmonary disease). Purine levels were actually modestly higher (approximately two-fold) in the induced samples, suggesting that the differences between $\mathrm{CF}$ and control could not be attributed to use of induction in the control population (data not shown).

To assess whether these findings were reproducible in airway secretions not accessible to expectoration, SMM obtained from the airways of explanted CF lungs at the time of transplantation was obtained. The pattern of purines in the SMM from 13 explanted CF lungs was similar to that observed in CF sputum, with proportionally higher levels of AMP and relatively low levels of adenosine (fig. 1b). Thus, the pattern of purines in $\mathrm{CF}$ secretions appeared to be independent of sample collection technique.

\section{Airway purines were elevated in BALF from children with airways disease and correlated with neutrophilic bronchitis} The present authors wished to determine whether the relationship between purines and airways disease could also be observed in children. Due to the frequent inability of children to expectorate sputum, airway secretions were obtained using bronchoalveolar lavage (BAL). BALF was prospectively collected and analysed from 25 children with $\mathrm{CF}$ and 12 non-CF subjects (disease controls; DC). Similar to the previous data, higher levels of both ATP and AMP were observed in CF BALF, compared with DC BALF, with AMP present at the highest absolute concentration (fig. 2a). Neither ADP nor adenosine levels differed among the groups.

While higher levels of ATP and AMP were observed in BALF from CF subjects, it could not be distinguished whether the differences were CF specific or reflected the higher levels of airway bacterial infection and/or neutrophilic bronchitis that characterise CF airways disease [34]. To determine the variables most closely correlated with purine levels, a multiple regression model was used to assess whether $\mathrm{CF}$ status, presence of airway infection $\left(>50,000\right.$ pathogens $\cdot \mathrm{mL}^{-1}$ on BALF culture) or neutrophil cell count best predicted ATP or 


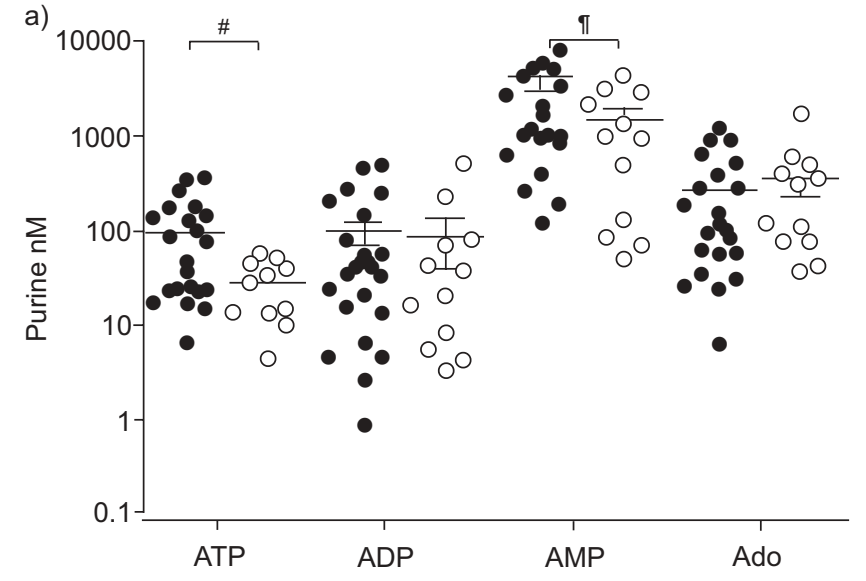

b)

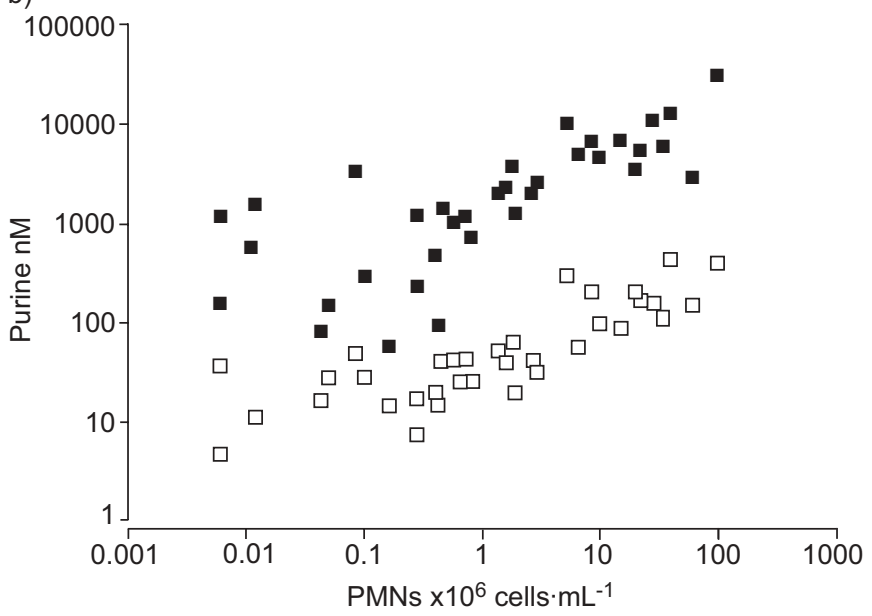

FIGURE 2. Purines in bronchoalveolar lavage fluid (BALF) from children were elevated in cystic fibrosis (CF) and correlated with neutrophilic airway inflammation. a) BALF was obtained from children with CF $(n=25 ; \bullet)$ and from disease controls $(n=12 ; 0)$ with other respiratory diseases. Measurement of purines in the supernatant of this BALF revealed elevated levels of adenosine triphosphate (ATP) and adenosine monophosphate (AMP) in the CF group. b) Statistically significant correlations were observed between polymorphonuclear neutrophil (PMN) counts and levels of both ATP ( $\square$ ) and AMP ( $\mathbf{\square})$ in BALF. ADP: adenosine diphosphate; Ado: adenosine. ${ }^{*}: p=0.0164 ;{ }^{\circ}: p=0.0300$. b) ATP: $r=0.81$, $p<0.0001$; AMP: $r=0.74, p<0.0001$

AMP levels in BALF. In the resulting model, only neutrophilic counts emerged as a significant predictor of ATP or AMP levels, with strong correlations to both ATP and AMP (fig. 2b). In contrast, neutrophil counts were not strongly correlated with either adenosine $(r=-0.22, p=0.19)$ or ADP $(r=0.25, p=0.14)$. Similar correlations were observed in the sputum samples between neutrophil counts and both ATP and AMP $(r=0.75$, $\mathrm{p}=0.0007$ and $\mathrm{r}=0.81, \mathrm{p}=0.0001$, respectively), although the results were limited by the small number of subjects from whom accurate cell counts could be obtained (three CF subjects and 14 controls). These data suggest that ATP and AMP are primarily biomarkers of neutrophilic inflammation.

\section{Airway purines correlated with lung function}

Since neutrophilic inflammation mediates many of the clinical manifestations of CF airways disease, including declines in lung function, the present authors tested for correlations between purine levels in BALF and lung function. Although significant correlations were observed between raw ATP and AMP values and lung function (data not shown), it was recognised that variable dilution of airway secretions in BALF could confound the relationship between BALF biomarkers and clinical outcomes. The current authors attempted to control for dilution using the BALF to serum urea ratio, a widely used albeit problematic dilution marker [35]. However, a correlation trend was observed between urea-based dilution factors and neutrophil counts $(r=-0.40, p=0.056)$, suggesting that BALF urea levels were artefactually increased in diseased airways, perhaps by increased efflux of urea through inflamed epithelia during lavage [35]. Therefore, an alternative method to control for dilution using ratios of ATP or AMP to adenosine was examined, since ratios are not affected by dilution. This method is based on the observation that both ATP and AMP correlate with markers of airways disease, whereas adenosine does not. As anticipated, significant correlations were observed for both ATP/adenosine and AMP/adenosine ratios with the percentage of neutrophils as dilution-independent markers of bronchitis (fig. 3a). Furthermore, significant correlations were also seen between the purine ratios and per cent predicted forced expiratory volume in one second (FEV1; fig. 3b). These data indicate that with appropriate methods to control for dilution, airway ATP and AMP levels correlate with a clinically relevant index of disease severity as expected for a biomarker of neutrophilic inflammation.

\section{ATP in EBC was correlated with airway inflammation}

While the present data demonstrated that the purines ATP and AMP are potential biomarkers of neutrophilic inflammation in children with $\mathrm{CF}$, risks and technical challenges associated with BAL limit clinical application. To determine whether airway purines could be measured using a simple and noninvasive method in children, the feasibility of detecting purines in EBC was explored.

In a pilot study, EBC was obtained from a small group of healthy and CF children (four in each group). Initial analyses using etheno-derivatisation and HPLC revealed that EBC purine concentrations were below the etheno-derivatisation detection threshold in most individuals (data not shown). As a more sensitive method, a highly sensitive and specific luminometry method was utilised, which is widely used to measure ATP levels in biological samples [25, 36, 37]. With luminometry, a signal above background was detected in each of the pilot EBC samples (mean \pm SE (range) $840 \pm 320$ (1572,428 ) arbitrary light units (ALU)). Consistent with the signal being ATP specific, the signal disappeared with sample preincubation for $30 \mathrm{~min}$ with 2 units $\cdot \mathrm{mL}^{-1}$ of the ATP degrading enzyme apyrase (post-treatment $15 \pm 5$ ALU; fig. 4a), but not in time-control incubations. Based on comparison to known standards, EBC ATP concentrations were in the low picomolar range, consistent with those measured in BALF and sputum after taking into account the $10^{3}-10^{4}$ (or higher) dilution of airway secretions in $\mathrm{EBC}[38,39]$. At this concentration range, reproducibility was modest, with within-sample coefficients of variation between $20-30 \%$. For this reason, EBC ATP levels were reported as the average of duplicate measures.

To further assess EBC ATP levels with the luminometric method, the pilot study was expanded to include EBCs 

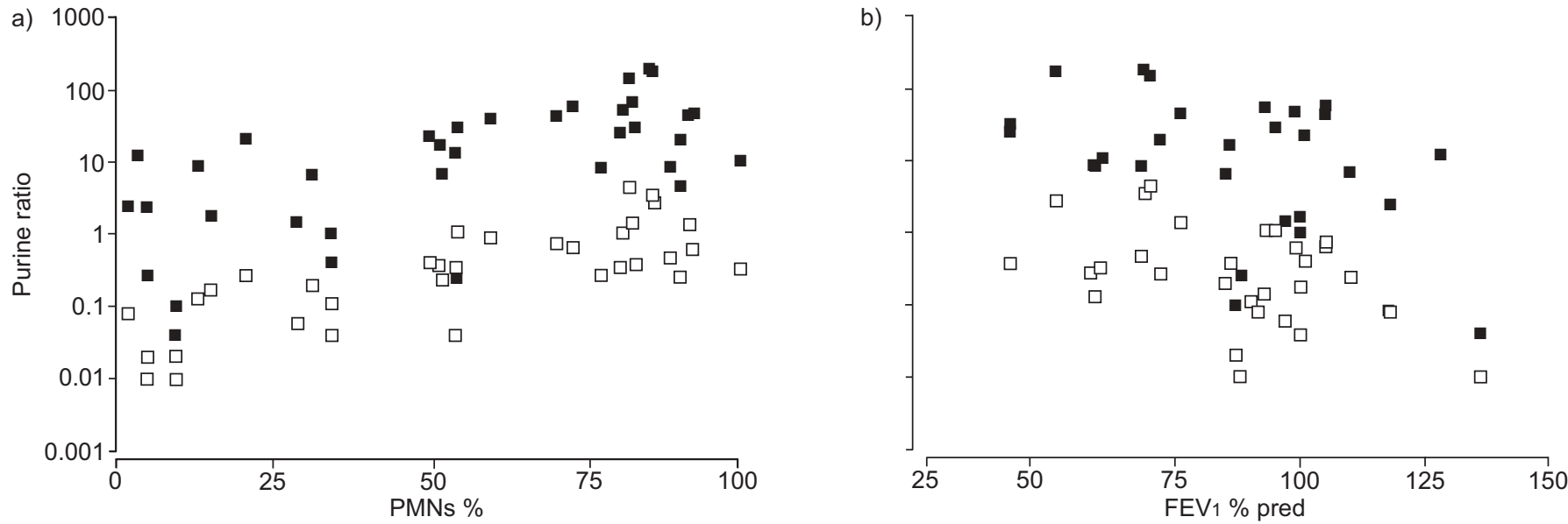

FIGURE 3. Purine ratios correlated with neutrophilic airway inflammation and lung function. Ratios of adenosine triphosphate (ATP) to adenosine (Ado; $\square$ ) and adenosine monophosphate (AMP) to Ado; - were calculated as markers of purine levels corrected for variable dilution of airway secretions in bronchoalveolar lavage fluid. a) Both ATP/Ado and AMP/Ado ratios were strongly correlated with percentage of polymorphonuclear neutrophils (PMNs) as a dilution-independent marker of inflammation. b) Purine ratios were compared with spirometry data available from a subset of subjects ( $n=28,20$ cystic fibrosis and eight disease controls). Both ATP/Ado and AMP/Ado ratios were negatively correlated with forced expiratory volume in one second (FEV1 \% predicted). a) ATP/Ado: $r=0.76, p<0.0001 ;$ AMP/Ado: $r=0.67, p<0.0001$. b) ATP/Ado: $r=-0.41, p=0.0335$; AMP/Ado: $r=-0.43, p=0.0240$.

collected from a total of 14 children with CF and 14 healthy controls during regular clinic visits. Consistent with the previous results, EBC ATP levels were elevated in children with CF compared with healthy controls (fig. 4b). Treatment of a $\mathrm{CF}$ exacerbation reduces neutrophilic airway inflammation [40]; thus, it was hypothesised that EBC ATP levels would drop during treatment. To explore this relationship, EBC was collected from 14 children with CF at the beginning and end of a course of intravenous antibiotics for a pulmonary exacerbation, including four CF subjects from the previous study. The mean \pm SD treatment duration was $21.6 \pm 3.3$ days, with antibiotics chosen by the treating physician. As expected, lung function improved after antibiotic treatment (mean \pm SD FEV1 $\%$ pred: $60.1 \pm 20.1 \%$ pre- versus $70.1 \pm 19.9 \%$ post-antibiotics, $\mathrm{p} \leqslant 0.01)$. This improvement was associated with a three-fold fall in EBC ATP levels (fig. 4c), consistent with previously observed changes in sputum markers of neutrophilic inflammation after treatment [40]. Although these data are preliminary, they do indicate that EBC ATP levels fit the pattern expected for a biomarker of neutrophilic inflammation.

\section{DISCUSSION}

Extracellular purines are the signalling molecules of purinergic signalling pathways that regulate airway defences, including mucociliary clearance and intraluminal inflammatory responses. In the present study, it was demonstrated that both the pattern and concentration of these purines have a strong relationship to CF airways inflammation. Analyses of secretions from CF subjects obtained as sputum, SMM and BALF provided a remarkably consistent picture in which AMP was the dominant purine, and both ATP and AMP were elevated in CF compared with control (table 2). This pattern of purines differed significantly from the relatively equal levels of AMP and adenosine observed in sputum from normal subjects, which in turn was similar to that reported in cultures of normal airway epithelia [32]. Although subjects with CF were primarily studied, the data suggest that the relationship between purines and neutrophilic inflammation is not CF specific. However, further study is necessary before the present findings can be confidently generalised to non-CF airways disease.

While the source of airway purines was not identified in the present study, the strong correlation between purine levels and neutrophil counts suggests that neutrophils may be a major contributor to purine levels in CF airway secretions. This conclusion is consistent with previous studies showing that activated neutrophils exhibit increased release of ATP and accumulate AMP [41]. However, purine release from necrotic or apoptotic cells cannot be excluded, nor can a significant contribution from inflamed airway epithelia or other inflammatory cells be ruled out. Although bacteria are another potential source of extracellular purines, it seems unlikely that bacteria significantly contributed to ATP release at levels commonly found in CF sputum $\left(\sim 10^{7} \cdot \mathrm{mL}^{-1}\right)$ [42]. The accumulation of AMP suggests a relative absence of $5^{\prime}$ nucleotidase metabolism of AMP to adenosine, a pattern which has been observed in isolated neutrophils (which lack 5 '-nucleotidase) but not airway epithelia [32, 43]. Therefore, the high levels of AMP in samples obtained from diseased airways suggest that neutrophils dominate the metabolic pattern of purine nucleotides on these airway surfaces.

Regardless of the underlying mechanism(s), the strong correlation with neutrophil counts indicates that airway levels of ATP and AMP are potential biomarkers of neutrophilic inflammation. The present data suggest that measurement of ATP in EBC may hold promise as a simple and noninvasive method to assess the degree of neutrophilic airway inflammation in children. Indeed, the approximately three-fold reduction in EBC ATP levels after treatment of CF pulmonary exacerbation parallels the reduction of neutrophil cell counts and IL-8 levels in sputum after similar treatment [40].

However, the current results also highlighted many of the known limitations to EBC analysis. The measured ATP 

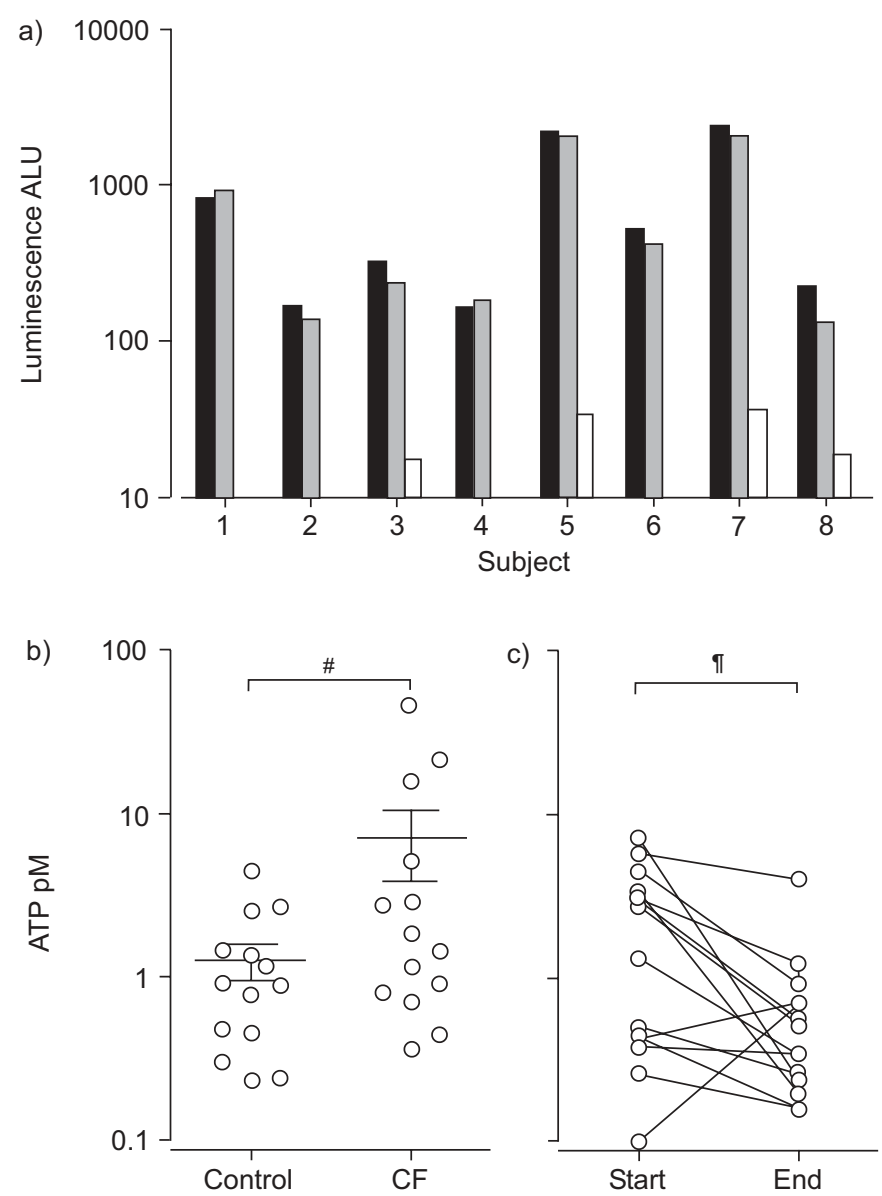

FIGURE 4. Adenosine triphosphate (ATP) in exhaled breath condensate (EBC) was elevated in cystic fibrosis (CF) and decreased with treatment of a CF exacerbation. ATP levels in EBC were measured by luminometry. a) Pilot EBC collections were obtained from four healthy controls (subjects 1-4) and four CF subjects (5-8). A luminescent signal was detected in all samples that was stable to incubation at $37^{\circ} \mathrm{C}$ for $30 \mathrm{~min}(\square)$, compared with samples kept at $0^{\circ} \mathrm{C}$ on ice $(\square)$, and disappeared after incubation with 2 units $\mathrm{mL}^{-1}$ of the ATP degrading enzyme apyrase ( $\square$ ). b) ATP levels were measured in EBC from CF ( $n=14)$ and healthy controls $(n=14)$. ATP was elevated in the CF group. c) ATP levels in EBC were measured at the start and end of a course of antibiotics to treat a pulmonary exacerbation in children with CF $(n=14)$. ATP levels were lower at the end of treatment. ALU: arbitrary light units. ${ }^{*}: p=0.0441 ; ": p=0.0175$.

concentrations were low and near the threshold for detection, suggesting that small technical artefacts or variations could significantly affect the results. Furthermore, the small volumes of EBC collected precluded measurement of both ATP and dilution markers. Consequently, systematic differences in sample dilutions among groups could not be assessed and could contribute to the observed differences. Therefore, further improvements in methodology are needed for more thorough evaluation of the clinical potential of EBC purine measurements.

While ATP and AMP emerged as potential biomarkers, similar findings were not observed for adenosine. While the levels of adenosine in BALF were similar to previously published values [27], the present authors did not observe the elevated levels of airway adenosine $[27,28]$ or correlation to disease
TABLE 2 Relative purine composition of airway secretions

\begin{tabular}{lrrrr} 
Sample & ATP & ADP & AMP & Ado \\
\hline $\begin{array}{l}\text { Healthy } \\
\quad \text { Sputum }\end{array}$ & $2.8 \pm 0.5$ & $9.8 \pm 1.9$ & $36.2 \pm 4.3$ & $51.2 \pm 4.9$ \\
$\begin{array}{l}\text { CF } \\
\quad \text { Sputum }\end{array}$ & & & & \\
SMM & $14.0 \pm 3.5$ & $1.5 \pm 0.7$ & $84.8 \pm 7.0$ & $5.7 \pm 3.0$ \\
$\quad$ BALF & $2.3 \pm 0.2$ & $2.7 \pm 0.8$ & $82.9 \pm 4.0$ & $12.0 \pm 3.9$ \\
\hline
\end{tabular}

Data are presented as mean \pm se relative percentages of each purine. The pattern of airway purines was similar in the various types of airway sample, but differed in healthy versus cystic fibrosis (CF) subjects. ATP: adenosine triphosphate; ADP: adenosine diphosphate; AMP: adenosine monophosphate; Ado: adenosine; SMM: supernatant of mucopurulent material; BALF: bronchoalveolar lavage fluid.

severity previously described for asthma [29]. This difference may reflect fundamental differences in airway pathophysiology between asthma and CF. CF is characterised by a pathogen- and neutrophil-dominated airway inflammation that differs significantly from the less cellular, eosinophil rich, and relatively sterile airway inflammation observed in asthma. These findings suggest that factors responsible for increased adenosine levels in asthma are not common in the neutrophilic bronchitis that characterises $\mathrm{CF}$.

In addition to exploring the potential of airway purines as biomarkers of inflammation, the present study also provides new insights into purinergic signalling pathways in airways disease. While it was not possible to accurately determine the dilution in the BALF samples, both the ATP and adenosine levels in BALF were consistent with previous reports [25, 27], and previous studies suggest that airway secretions in BALF were probably diluted 10-100-fold [35]. Given this level of dilution, in situ ATP levels would be above the median effective concentration (EC50) for the $\mathrm{P}_{2} \mathrm{Y}_{2}$ ATP receptor in airway epithelia $(0.24 \mu \mathrm{M})$ [44] and approach the levels needed to activate $\mathrm{P}_{2} \mathrm{Y}_{2}$ receptors in inflammatory cells $[13,45]$. These considerations suggest that ATP-mediated purinergic signalling may be adaptively increased in bacterially infected airways, leading to higher mucociliary clearance and greater activation of inflammatory cells. Similarly, the average adenosine levels in both healthy and $\mathrm{CF}$ airways are predicted to be above the EC50 values of the A1 and $\mathrm{A} 2 \mathrm{a}$ adenosine receptors $(0.31$ and $0.73 \mu \mathrm{M}$, respectively) found on inflammatory cells [46,47], although somewhat lower than the EC50 of the dominant adenosine receptor (A2b) expressed in airway epithelia [32]. As adenosine levels did not correlate with markers of disease, the present authors would not anticipate that adenosine-mediated signalling would increase with neutrophilic inflammation, and the increased ATP to adenosine ratio might favour a more pro-inflammatory environment [20]. However, recently published evidence suggests that AMP may serve as an adenosine receptor agonist, either directly [48] or after conversion to adenosine at the epithelial surface [49]. Thus, increased adenosine receptor signalling, mediated through AMP, could modulate increased inflammation.

In conclusion, the present study demonstrates that purines recovered in airway secretions are biomarkers of neutrophilic 
airway inflammation and are elevated in cystic fibrosis airways disease. The results suggest that measurement of purines in exhaled breath condensate may be useful as a noninvasive method of assessing airway inflammation, although further refinements are necessary.

\section{ACKNOWLEDGEMENTS}

The authors wish to thank C. van Heusden (University of North Carolina at Chapel Hill, Chapel Hill, NC, USA) for assistance measuring purine levels in airway samples.

\section{REFERENCES}

1 Burnstock G. Purinergic nerves. Pharmacol Rev 1972; 24: 509-581.

2 Burnstock G. Purinergic signalling. Br J Pharmacol 2006; 147: Suppl. 1, S172-S181.

3 Dubyak GR, el-Moatassim C. Signal transduction via P2purinergic receptors for extracellular ATP and other nucleotides. Am J Physiol 1993; 265: C577-C606.

4 Ahmad S, Ahmad A, McConville G, et al. Lung epithelial cells release ATP during ozone exposure: signaling for cell survival. Free Radic Biol Med 2005; 39: 213-226.

5 Smith CP, Vemulakonda VM, Kiss S, Boone TB, Somogyi GT. Enhanced ATP release from rat bladder urothelium during chronic bladder inflammation: effect of botulinum toxin A. Neurochem Int 2005; 47: 291-297.

6 Kunzelmann K, Scheidt K, Scharf B, et al. Flagellin of Pseudomonas aeruginosa inhibits $\mathrm{Na}+$ transport in airway epithelia. FASEB J 2006; 20: 545-546.

7 Chen Y, Shukla A, Namiki S, Insel PA, Junger WG. A putative osmoreceptor system that controls neutrophil function through the release of ATP, its conversion to adenosine, and activation of A2 adenosine and P2 receptors. J Leukoc Biol 2004; 76: 245-253.

8 Madara JL, Patapoff TW, Gillece-Castro B, et al. 5'adenosine monophosphate is the neutrophil-derived paracrine factor that elicits chloride secretion from T84 intestinal epithelial cell monolayers. J Clin Invest 1993; 91: 2320-2325.

9 Sperlágh B, Haskó G, Németh Z, Vizi ES. ATP released by LPS increases nitric oxide production in raw 264.7 macrophage cell line via $\mathrm{P} 2 \mathrm{Z} / \mathrm{P} 2 \mathrm{X} 7$ receptors. Neurochem Int 1998; 33: 209-215.

10 Chen Y, Corriden R, Inoue Y, et al. ATP release guides neutrophil chemotaxis via P2Y2 and A3 receptors. Science 2006; 314: 1792-1795.

11 Lennon PF, Taylor CT, Stahl GL, Colgan SP. Neutrophilderived 5'-adenosine monophosphate promotes endothelial barrier function via CD73-mediated conversion to adenosine and endothelial A2B receptor activation. J Exp Med 1998; 188: 1433-1443.

12 Picher M, Burch LH, Boucher RC. Metabolism of P2 receptor agonists in human airways: implications for mucociliary clearance and cystic fibrosis. J Biol Chem 2004; 279: 20234-20241.

13 Meshki J, Tuluc F, Bredetean O, Ding Z, Kunapuli SP. Molecular mechanism of nucleotide-induced primary granule release in human neutrophils: role for the P2Y2 receptor. Am J Physiol Cell Physiol 2004; 286: C264-C271.
14 Cockcroft S, Stutchfield J. The receptors for ATP and fMetLeuPhe are independently coupled to phospholipases $\mathrm{C}$ and A2 via G-protein(s). Relationship between phospholipase C and A2 activation and exocytosis in HL60 cells and human neutrophils. Biochem J 1989; 263: 715-723.

15 Verghese MW, Kneisler TB, Boucheron JA. P2U agonists induce chemotaxis and actin polymerization in human neutrophils and differentiated HL60 cells. J Biol Chem 1996; 271: 15597-15601.

16 Ferrari D, Chiozzi P, Falzoni S, et al. Extracellular ATP triggers IL-1 $\beta$ release by activating the purinergic P2Z receptor of human macrophages. J Immunol 1997; 159: 1451-1458.

17 Di Virgilio F, Chiozzi P, Ferrari D, et al. Nucleotide receptors: an emerging family of regulatory molecules in blood cells. Blood 2001; 97: 587-600.

18 Schmid-Antomarchi H, Schmid-Alliana A, Romey G, et al. Extracellular ATP and UTP control the generation of reactive oxygen intermediates in human macrophages through the opening of a charybdotoxin-sensitive Ca2+dependent K+ channel. J Immunol 1997; 159: 6209-6215.

19 Aga M, Watters JJ, Pfeiffer ZA, Wiepz GJ, Sommer JA, Bertics PJ. Evidence for nucleotide receptor modulation of cross talk between MAP kinase and NF- $\kappa B$ signaling pathways in murine RAW 264.7 macrophages. Am J Physiol Cell Physiol 2004; 286: C923-C930.

20 Bours MJ, Swennen EL, Di Virgilio F, Cronstein BN, Dagnelie PC. Adenosine 5'-triphosphate and adenosine as endogenous signaling molecules in immunity and inflammation. Pharmacol Ther 2006; 112: 358-404.

21 Geary C, Akinbi H, Korfhagen T, Fabre JE, Boucher R, Rice W. Increased susceptibility of purinergic receptordeficient mice to lung infection with Pseudomonas aeruginosa. Am J Physiol Lung Cell Mol Physiol 2005; 289: L890-L895.

22 Ma B, Blackburn MR, Lee CG, et al. Adenosine metabolism and murine strain-specific IL-4-induced inflammation, emphysema, and fibrosis. J Clin Invest 2006; 116: 1274-1283.

23 Chunn JL, Molina JG, Mi T, Xia Y, Kellems RE, Blackburn MR. Adenosine-dependent pulmonary fibrosis in adenosine deaminase-deficient mice. J Immunol 2005; 175: 1937-1946.

24 Chunn JL, Young HW, Banerjee SK, Colasurdo GN, Blackburn MR. Adenosine-dependent airway inflammation and hyperresponsiveness in partially adenosine deaminase-deficient mice. J Immunol 2001; 167: 4676-4685.

25 Donaldson SH, Lazarowski ER, Picher M, Knowles MR, Stutts MJ, Boucher RC. Basal nucleotide levels, release, and metabolism in normal and cystic fibrosis airways. Mol Med 2000; 6: 969-982.

26 Lader AS, Prat AG, Jackson GR Jr, et al. Increased circulating levels of plasma ATP in cystic fibrosis patients. Clin Physiol 2000; 20: 348-353.

27 Driver AG, Kukoly CA, Ali S, Mustafa SJ. Adenosine in bronchoalveolar lavage fluid in asthma. Am Rev Respir Dis 1993; 148: 91-97.

28 Huszár E, Vass G, Vizi E, et al. Adenosine in exhaled breath condensate in healthy volunteers and in patients with asthma. Eur Respir J 2002; 20: 1393-1398.

29 Csoma Z, Huszár E, Vizi E, et al. Adenosine level in exhaled breath increases during exercise-induced bronchoconstriction. Eur Respir J 2005; 25: 873-878. 
30 Alexis NE, Soukup J, Nierkens S, Becker S. Association between airway hyperreactivity and bronchial macrophage dysfunction in individuals with mild asthma. Am J Physiol Lung Cell Mol Physiol 2001; 280: L369-L375.

31 Ribeiro CM, Paradiso AM, Carew MA, Shears SB, Boucher RC. Cystic fibrosis airway epithelial Ca2+ i signaling: the mechanism for the larger agonist-mediated $\mathrm{Ca} 2+\mathrm{i}$ signals in human cystic fibrosis airway epithelia. J Biol Chem 2005; 280: 10202-10209.

32 Lazarowski ER, Tarran R, Grubb BR, van Heusden CA, Okada S, Boucher RC. Nucleotide release provides a mechanism for airway surface liquid homeostasis. J Biol Chem 2004; 279: 36855-36864.

33 Watt WC, Lazarowski ER, Boucher RC. Cystic fibrosis transmembrane regulator-independent release of ATP. Its implications for the regulation of $\mathrm{P} 2 \mathrm{Y} 2$ receptors in airway epithelia. J Biol Chem 1998; 273: 14053-14058.

34 Muhlebach MS, Noah TL. Endotoxin activity and inflammatory markers in the airways of young patients with cystic fibrosis. Am J Respir Crit Care Med 2002; 165: 911-915.

35 Haslam PL, Baughman RP. Report of ERS Task Force: guidelines for measurement of acellular components and standardization of BAL. Eur Respir J 1999; 14: 245-248.

36 Douillet CD, Suy S, Zarzaur BL, et al. Measurement of free and bound fractions of extracellular ATP in biological solutions using bioluminescence. Luminescence 2005; 20 : 435-441.

37 Sprague RS, Ellsworth ML, Stephenson AH, Kleinhenz ME, Lonigro AJ. Deformation-induced ATP release from red blood cells requires CFTR activity. Am J Physiol 1998; 275: H1726-H1732.

38 Effros RM, Biller J, Foss B, et al. A simple method for estimating respiratory solute dilution in exhaled breath condensates. Am J Respir Crit Care Med 2003; 168: 1500-1505.

39 Effros RM, Hoagland KW, Bosbous M, et al. Dilution of respiratory solutes in exhaled condensates. Am J Respir Crit Care Med 2002; 165: 663-669.
40 Ordoñez CL, Henig NR, Mayer-Hamblett N, et al. Inflammatory and microbiologic markers in induced sputum after intravenous antibiotics in cystic fibrosis. Am J Respir Crit Care Med 2003; 168: 1471-1475.

41 Eltzschig HK, Eckle T, Mager A, et al. ATP release from activated neutrophils occurs via connexin 43 and modulates adenosine-dependent endothelial cell function. Circ Res 2006; 99: 1100-1108.

42 Crane JK, Olson RA, Jones HM, Duffey ME. Release of ATP during host cell killing by enteropathogenic E. coli and its role as a secretory mediator. Am J Physiol Gastrointest Liver Physiol 2002; 283: G74-G86.

43 Lazarowski ER, Boucher RC, Harden TK. Mechanisms of release of nucleotides and integration of their action as P2X- and P2Y-receptor activating molecules. Mol Pharmacol 2003; 64: 785-795.

44 Lazarowski ER, Watt WC, Stutts MJ, Boucher RC, Harden TK. Pharmacological selectivity of the cloned human P2U-purinoceptor: potent activation by diadenosine tetraphosphate. Br J Pharmacol 1995; 116: 1619-1627.

45 Wang L, Jacobsen SE, Bengtsson A, Erlinge D. P2 receptor mRNA expression profiles in human lymphocytes, monocytes and CD34+ stem and progenitor cells. BMC Immunol 2004; 5: 16.

46 Fredholm BB, Irenius E, Kull B, Schulte G. Comparison of the potency of adenosine as an agonist at human adenosine receptors expressed in Chinese hamster ovary cells. Biochem Pharmacol 2001; 61: 443-448.

47 Marone G, Petracca R, Vigorita S. Adenosine receptors on human inflammatory cells. Int Arch Allergy Appl Immunol 1985; 77: 259-263.

48 Gao N, Hu HZ, Liu S, Gao C, Xia Y, Wood JD. Stimulation of adenosine $\mathrm{A} 1$ and $\mathrm{A} 2 \mathrm{~A}$ receptors by $\mathrm{AMP}$ in the submucosal plexus of guinea pig small intestine. Am J Physiol Gastrointest Liver Physiol 2007; 292: G492-G500.

49 Sitaraman SV, Merlin D, Wang L, et al. Neutrophilepithelial crosstalk at the intestinal lumenal surface mediated by reciprocal secretion of adenosine and IL-6. J Clin Invest 2001; 107: 861-869. 